\title{
Difficulties in the diagnosis of four repeats (4R) tauopathic parkinsonian syndromes
}

\author{
Piotr Alster ${ }^{a, *}$, Ewa Krzyżanowska ${ }^{b}$, Dariusz Koziorowski ${ }^{a}$, \\ Stanisław Szlufik ${ }^{a}$, Dorota Różański ${ }^{a}$, Joanna Noskowska ${ }^{b}$, \\ Justyna Mianowicz ${ }^{b}$, Adrian Michno ${ }^{b}$, Leszek Królicki ${ }^{c}$, Andrzej Friedman ${ }^{a}$ \\ ${ }^{a}$ Department of Neurology, Medical University of Warsaw, Poland \\ b Brodno Mazovian Hospital, Poland \\ ${ }^{\mathrm{c}}$ Department of Nuclear Medicine, Medical University of Warsaw, Poland
}

\section{A R T I C L E I N F O}

Article history:

Received 25 January 2018

Accepted 19 June 2018

Available online 3 July 2018

\section{Keywords:}

Tauopathy

Atypical parkinsonism

PSP

CBD

CBS

\begin{abstract}
A B S T R A C T
Corticobasal Degeneration Degeneration (CBD) and Progressive Supranuclear Palsy (PSP) are types of four repeats (4R) tauopathies, which are associated to parkinsonian syndromes. The aim of the work is to analyze cases of patients of the Department of Neurology, overlapping of syndromes related to both pathologies and to show that most likely CBS and PSP are not lineary related to their commonly associated syndromes i.e. adequately corticobasal syndromes and progressive supranuclear palsy syndromes. In the context of each patient factors in favor of most likely CBS, PSP or both diseases are discussed and analyzed using contemporary criteria. This work discusses multidimensional aspect of the examination of five patient aged 64 to $83-4$ females and 1 male with $4 \mathrm{R}$ tauopathies and difficulties in distinguishing both diseases. The duration of the disease varied from 1 to 5 years. Each patient after neurological examination was assessed using magnetic resonance imaging (MRI) and psychological test. Examination of all patients was extended using single photon emission computer tomography (SPECT) to reveal the usefulness of this tool in differentiation of diseases was done. The outcome of this examination was verified with prior clinical manifestation of patients and morphological abnormalities in magnetic resonance imaging. Autopsies were not conducted.
\end{abstract}

(c) 2018 Polish Neurological Society. Published by Elsevier Sp. z o.o. All rights reserved.

\section{Introduction}

Corticobasal degeneration (CBD) and progressive supranuclear palsy (PSP) belong to atypical parkinsonisms (APS) which due to their histopathology are defined as primary tauopathies [1-7]. In the classification of tauopathies PSP and corticobasal syndrome (CBS) are most commonly associated with tau proteins containing four repeats (4R) of approximately 32 aminoacids in the microtubule binding domain [1-3]. Nevertheless regarding the fact that CBS may be related to $4 R$ pathologies such as CBD and PSP, but also Alzheimer's disease (AD) and Pick's disease which are associated with three repeats tauopathies (3R), it should be stressed that CBS may be a clinical manifestation of either $3 R$ or $4 R$ [7]. It should also be highlighted that $\mathrm{AD}$ may be related to accumulation of both

\footnotetext{
* Corresponding author at: Department of Neurology, Medical University of Warsaw, Kondratowicza 8, 03-242 Warsaw, Poland.

E-mail address: Piotr.Alster@gmail.com (P. Alster).

https://doi.org/10.1016/j.pjnns.2018.06.002

0028-3843/○ 2018 Polish Neurological Society. Published by Elsevier Sp. z o.o. All rights reserved.
} 
$3 R$ and $4 R$ [7]. This is one of the aspects allowing neuropathological differentiation between CBD/PSP and frontotemporal lobar degeneration, which is related with three repeats (3R). PSP and less common CBD were described in 1960s (PSP in 1964, CBD in 1968) [4-7] as neurodegenerative diseases with relatively short duration and rather difficult differentiation from each other and other extrapyramidal disorders [4]. PSP and CBD due to their similar symptomatology and pathology are often mistaken with each other. Often patients have symptoms characteristic for both diseases.

The aim of the study was to discuss whether strict differentiation of most likely CBS and PSP is justified in all cases. Clinical presentations were correlated with mental dysfunction and neuroimaging.

\section{Material and methods}

Five patients with clinical symptoms combining CBS and PSP were assessed. In this study generally accepted criteria of diagnosis of CBD and PSP were used [5,6] (Tables 1 and 2). The presence and stage of ocular motor dysfunction, postural instability, akinesia and cognitive dysfunction were used as basic criteria of the most common subtype of PSP - Richardson syndrome. The certainty of diagnosis was based on the intensity of symptoms. (Table 1). CBD was examined by assessing the presence and stage of apraxia, alien limb phenomena, cortical sensory loss, cognitive impairment, behavioral changes, and aphasia, asymmetric onset of levodopa-resistant parkinsonism, dystonia, and myoclonus. The more symptoms were present in the clinical examination, the more certain the diagnosis was (Table 2). Symptoms of both diseases were observed among all patients demonstrated in the study. The inclusion and exclusion criteria of both diseases were assessed. In this work 5 case studies of patients with insufficient symptomatology to diagnose either CBD or PSP are demonstrated (Table S1). All of these patients were examined by neurologists experienced in movement disorders
- (AF, DK). Psychological examinations were performed using Montreal Cognitive Assessment (MoCA) or Mini Mental State Examination (MMSE) and Frontal Assessment Battery (FAB). Neuroimaging was performed using magnetic resonance imaging (MRI) and single photon emission computer tomography (SPECT). The project was approved by the Ethical Committee of the Medical University of Warsaw - AKBE243/ 2016.

\section{Study}

\subsection{Patient \#1}

A 82-year-old female patient was admitted to the Department of Neurology in November of 2016. Since 2012, when she was 78 years old, the patient's family observed slowness of speech, psychomotor slowness and falls. At the time of the examination the patient presented facial masking, oculomotor apraxia, paresis of the right upper and lower limb. Bradykinesia was more visible on the right side of the body than on the left. Patient presented more severe bradykinesia in lower limbs than in upper ones, general psychomotor slowness, retropulsion and lack of balancing during gait. The patient complained of deterioration of handwriting, memory deficits, symptoms of orthostatic hypotension, constipation, dysuria and abnormal sweating. Logopedic examination demonstrated bradylalic non-fluent speech. The patient had difficulties in initiation of speech. Nasal speech, dysprosody and echolalia could also be observed. Psychological examination revealed dementia of moderate intensity with apraxia, impaired executive functions, impaired attention, deteriorated learning and memory abilities. Posturography confirmed static and dynamic imbalance. MRI revealed slightly reduced volume of the midbrain, medianus dimension (anterior-posterior) in the midline approx. $14 \mathrm{~mm}$ and slightly concave top surface, the picture was not evident. SPECT highlighted irregular distribution of the radiopharmaceutic hexamethylpropyleneamine

Table 1 - Corticobasal degeneration - criteria* [5].

\begin{tabular}{|c|c|c|c|c|c|}
\hline Diagnosis/symptoms & $\begin{array}{l}\text { Limb rigidity } \\
\text { of akinesia }\end{array}$ & $\begin{array}{l}\text { Limb } \\
\text { dystonia }\end{array}$ & $\begin{array}{l}\text { Limb } \\
\text { myoclonus }\end{array}$ & $\begin{array}{l}\text { - Orobuccal or limb apraxia } \\
\text { - Cortical sensory deficit } \\
\text { - Alien limb phenomena } \\
\text { (more than simple } \\
\text { levitation) }\end{array}$ & Other \\
\hline $\begin{array}{l}\text { Possible CBS } \\
\text { (clinical presentation } \\
\text { may be symmetric) }\end{array}$ & $\begin{array}{l}1 \text { of mentioned } \\
\text { above symptoms }\end{array}$ & & & $\begin{array}{l}1 \text { of mentioned above } \\
\text { symptoms }\end{array}$ & - \\
\hline $\begin{array}{l}\text { Probable CBS } \\
\text { (asymmetric) }\end{array}$ & $\begin{array}{l}2 \text { of mentioned } \\
\text { above symptoms }\end{array}$ & & & $\begin{array}{l}2 \text { of mentioned above } \\
\text { symptoms }\end{array}$ & 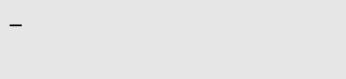 \\
\hline CBD-PSP & $*$ & $*$ & * & * & $\begin{array}{l}\text { * }-3 \text { of: } \\
\text { (a) axial or asymmetric } \\
\text { limb rigidity or akinesia } \\
\text { (b) postural instability } \\
\text { or falls } \\
\text { (c) urinary incontinence } \\
\text { behavioral changes } \\
\text { (d) supranuclear vertical } \\
\text { gaze palsy or decreased } \\
\text { velocity of vertical saccades }\end{array}$ \\
\hline
\end{tabular}




\begin{tabular}{|c|c|c|c|c|}
\hline Level of certainty & $\begin{array}{l}\text { Ocular motor } \\
\text { dysfunction }\end{array}$ & Postural instability & Akinesia & Cognitive dysfunction \\
\hline 1 (highest) & $\begin{array}{l}\text { Vertical supranuclear } \\
\text { gaze palsy }\end{array}$ & $\begin{array}{l}\text { Repeated unprovoked } \\
\text { falls within } 3 \text { years }\end{array}$ & $\begin{array}{l}\text { Progressive gait freezing } \\
\text { within } 3 \text { years }\end{array}$ & $\begin{array}{l}\text { Speech/language disorder } \\
\text { i.e. nonfluent/agrammatic } \\
\text { variant of primary progressive } \\
\text { aphasia or progressive apraxia } \\
\text { of speech }\end{array}$ \\
\hline 2 & $\begin{array}{l}\text { Slow velocity of vertical } \\
\text { saccades }\end{array}$ & $\begin{array}{l}\text { Tendency to fall on the } \\
\text { pull-test within } 3 \text { years }\end{array}$ & $\begin{array}{l}\text { Parkinsonism, akinetic- } \\
\text { rigid predominantly axial } \\
\text { and levodopa resistant }\end{array}$ & $\begin{array}{l}\text { Frontal cognitive/behavioral } \\
\text { presentation }\end{array}$ \\
\hline 3 (lowest) & $\begin{array}{l}\text { Frequent macro square } \\
\text { wave jerks or "eyelid } \\
\text { opening apraxia" }\end{array}$ & $\begin{array}{l}\text { More than two steps } \\
\text { backward on the } \\
\text { pull-test within } 3 \text { years }\end{array}$ & $\begin{array}{l}\text { Parkinsonism, with tremor } \\
\text { and/or asymmetric and/or } \\
\text { levodopa responsive }\end{array}$ & Corticobasal syndrome \\
\hline
\end{tabular}

Based on Ref. [6].

oxime (HMPAO) with a decreased accumulation of 99mTcHMPAO within the anterior part of parietal lobe and corpus callosum on the left side. Significant decrease in the accumulation was observed within the basal ganglia, nucleus caudatus on the left side, cerebellum on the left side and thalamus bilaterally. Pattern of HMPAO distribution was interpreted as resembling CBS. The patient also suffered due to hypertension and diabetes type 2 (Image 1).

\subsection{MRI - decreased volume of midbrain - Patient \#1}

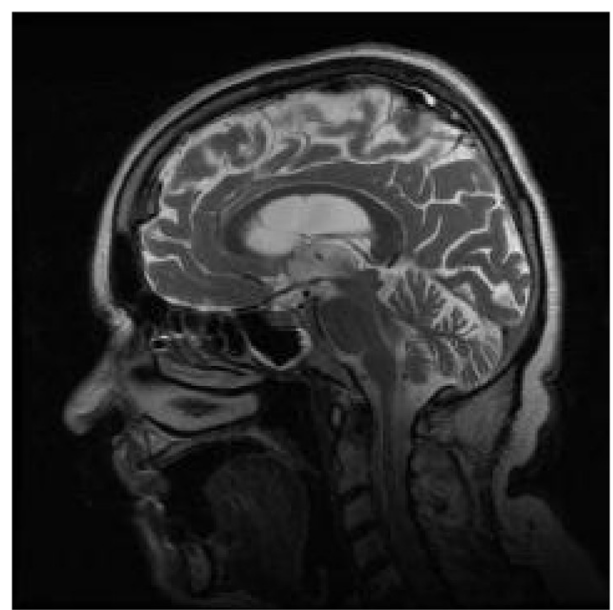

1.2. SPECT - asymmetric decrease in blood flow - Patient \#1

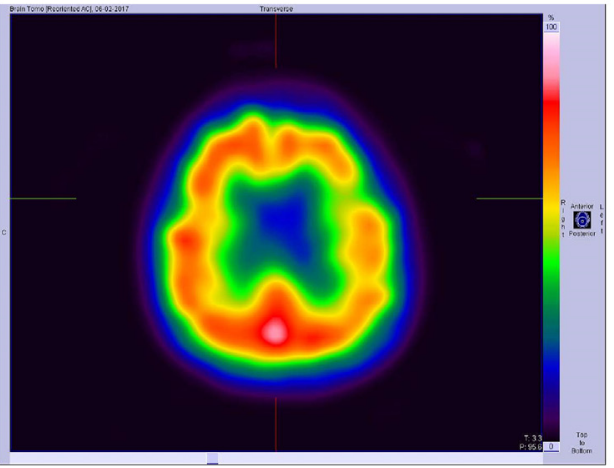

Image 1 - (1.1) MRI - decreased volume of midbrain - Patient \#1. (1.2) SPECT - asymmetric decrease in blood flow Patient \#1.

\subsection{Patient \#2}

A 65-year-old female patient presented five year history of parkinsonian syndrome which began with bradykinesia at the age of 60 . After five years intensive rigidity on the right side appeared. More brisk reflexes and Babinski sign on the right could also be observed. Psychological examination demonstrated disturbed episodic, operational and direct memory and deficits in executive functions. Logopedic examination revealed bradylalia and non-fluent speech. MRI showed disseminated small ischemic regions. Cortical atrophy within the left parietal lobe was observed. MRI also indicated cerebellar atrophy. SPECT imaging revealed limited accumulation of radiotracer within the frontal lobe, mainly on the left side. The patient did not suffer due to any other chronic illness.

\subsection{Patient \#3}

A 64-year-old male was admitted to the Department of Neurology due to progressive speech disorders, cognitive impairment, bradykinesia, paresis of right upper limb and balance abnormalities. First symptoms appeared at the age of 59. In neurological examination the patient showed involuntary movement of the right upper limb. The speech was nonfluent due to dysarthria. The patient presented cognitive impairment, bradykinesia and limitations in movements of eyeballs in all directions, deliberative symptoms, bilateral pyramidal syndrome, arrhythmia disturbance and widened asymmetric gait. The logopedic examination confirmed aphasia and dysarthria. The neuropsychological study was dominated by linguistic and frontal disorders. In stabilometric tests gait imbalance was observed. After administration of $200 \mathrm{mg}$ of levodopa no effect on the neurological condition was observed. MR scans revealed asymmetric cortical atrophy mainly in the left lateral sulcus. Atrophy of mesencephalon was also indicated. SPECT examination revealed reduced accumulation of the radiopharmaceutic on the left side within frontal superior and medium gyrus, temporal superior gyrus, Rolandic operculum and insula. The image indicated that the neurodegenerative deterioration might be due to CBS or PSP. The patient also suffered due to vascular changes in the central nervous system, acute pancreatitis in the past. He was also addicted to alcohol. 


\subsection{Patient \#4}

A 65-year-old female patient was admitted to the Department of Neurology for further diagnosis of postural instability with falls, bradykinesia and rigidity of left limbs. First symptoms occurred at the age of 63. In neurological examination cognitive impairment, dysarthria, hypomimia, bradykinesia, hypokinesia more severe in the left limbs, massive rigidity of the left limbs, postural disorders, gait dysfunction, clonuses, bilateral Babinski symptom were observed. Neuropsychological examination revealed dementia. MRI showed small disseminated vascular changes. Moreover signs of marginal cortical degeneration were observed. In SPECT 99mTc-HMPAO examination a decrease in the accumulation of the radiotracer was observed in the occipital lobe, insula and globus pallidus on the left side. Simultaneously normal perfusion on both sides of the thalamus was observed. The patient suffered from cognitive impairments, verbal disorders and hypertension and cervical discopathy.

\subsection{Patient \#5}

A 73-year-old female was admitted to the Department of Neurology for extended diagnosis due to involuntary movements within the face, gait disorders, slowness of movement and deteriorating cognitive impairments, which began two years before hospitalization and were followed after one year by falls. The first symptoms appeared at the age of 71 . In neurological examination hypokinesia, bradykinesia, rigidity were more intensive on the right side, dystonia of the right foot, cramp of left half of the face, dysarthria, abnormalities in postural reflexes and limited vertical eye movement looking down were found. MRI showed humming bird sign. No other abnormalities in examination of mesencephalon and pons where detected. SPECT 99mTc-HMPAO revealed decreased accumulation within the left thalamus, left inferior parietal gyrus, right cingulate and paracingulate, right thalamus, left superior temporal gyrus, parietal lobe and left middle cingulate and paracingulate. The patient also suffered due to depression, hypertension and hypercholesterolemia.

\section{Discussion}

The present case studies indicate that CBD and PSP share similar symptoms. Therefore, their clinical differentiation may be challenging for physicians. Both disorders share some symptoms such as parkinsonian syndrome, aphasia and progressive cognitive impairments. Other symptoms such as asymmetry of symptoms may be subjective or could not differentiate the diseases. These factors create a controversy, whether to differentiate diseases based on clinical symptomatology or to diagnose a disease as most likely CBS variant PSP or most likely PSP variant CBS. Additionally symptoms such as cognitive impairment or progressive dementia not only can be associated with PSP and CBS, but also may be due to vascular disorders which creates another difficulty in examination and proper diagnosis.

In a study, in which 39 cases were analyzed, CBS was associated with CBD pathology only in $55 \%$ of cases, in $20.5 \%$ with PSP pathology and in $7.2 \%$ with Pick's disease pathology [7]. This may possibly lead to considering the clinical picture of CBS as a marker of tauopathies. On the other hand both CBS and PSP tend to have different histopathological basis. Another study analyzed how CBS was associated with various pathologies [8]. It showed that in contrast with widespread changes in AD pathology, PSP and CBD pathology with clinical CBS was related preferably to more focal atrophies, predominantly within the premotor area.

It is stressed that unlike PSP, where clinical picture of PSPRS is highly predictive for PSP pathology, no clinical symptom is predictive of CBD [9]. Cases of corticobasal syndrome related to $A D$ pathology have also been recently presented [10]. Among symptoms of corticobasal syndrome related to $\mathrm{AD}$, authors of that article present limb myoclonus, dressing apraxia, memory loss, lower Mini Mental State Examination results, cortical sensory loss, visuospatial difficulties, hemisensory neglect and absence of limb rigidity. The study stresses longer duration of the disease compared with CBS associated with CBD pathology.

In CBS and PSP syndromes/Richardson's syndromes (PSPS/ RS) the clinical pictures quite often do not correlate with respective CBD and PSP pathologies [11]. Though one of the studies showed greater tau load in mid-frontal and inferiorparietal cortices in patients with PSP pathology and CBS clinical manifestation (PSP-CBS) than PSP pathology and PSPRS. The same study presented similar severity of those pathologies. It should also be highlighted that PSP-CBS is one of many clinical presentations of PSP pathology such as PSP parkinsonism (PSP-P), PSP with pure akinesia and gait freezing (PSP-PAGF), PSP with behavioral variant of frontotemporal dementia (PSP-bvFTD) and PSP with primary nonfluent afasia (PSP-nfaPNFA) [12] (Table S2). Moreover, vascular pathology mimicking PSP with predominant lower body involvement had been described [13]. Among the types mentioned above, PSP-RS with postural instability, supranuclear gaze palsy and frontal dysfunction and PSP-P associated with asymmetric onset, tremor and limited response to levodopa are the most common. It should also be stressed that some of the patients who never presented ocular motor symptoms, are post mortem diagnosed with PSP [14]. Superior cerebellar peduncle, subthalamic nucleus atrophy and midbrain atrophy also known as humming bird sign in MRI may be useful in differential diagnosis. However recent research shows that midbrain atrophy is correlated with clinical manifestation of PSP, patients with PSP pathology and non-PSP symptomatology tend to not have reduced volume of midbrain [15]. Therefore researches also stress that humming bird sign should not be perceived as an indicator of PSP pathology $[15,16]$.

Histopathological differentiation criteria had been shown in various studies [17-23]. According to those perspectives, PSP is preferably associated with tau-positive neurofibrillary tangles (NFT), coiled bodies, threads and tufted astrocytes [17]. The localization of NFT in PSP is related to subcortical and brainstem nuclei and cerebellar dentate nucleus $[17,18]$. Criteria specify the presence of pathological accumulation of abnormally phosphorylated microtubule-associated domain into filamentous deposits. In CBD, tau pathology affects both neurons and glia and the typical findings include pretangles, 
ballooned neurons, and astrocytic plaques. As an interesting factor may be interpreted "minimal pathological features of CBD", among which can be mentioned cortical and striatal tau-positive neuronal and glial lesions [17]. This may be a striking feature in the context of the growing number of various clinical manifestations of $C B D$, which were premortem interpreted as PSP. A case study published in 2000 described a Japanese woman with gradually developing parkinsonism [23]. Gaze palsy, dementia and dystonia were observed. Clinical manifestation was interpreted as PSP. The woman died at the age of 71 of respiratory failure. Histopathological examination of her brain revealed the presence of astrocytic plaques and swollen achromatic neurons that supported the diagnosis of CBD [23]. It is stressed that due to the similarities observed in the neuropathology of CBD and PSP, more detailed analysis aiming to distinguish abnormal tau inclusions may be necessary [21]. This brings to a point where differentiation between tufted astrocyted and astrocytic plaques becomes relevant [17]. These methods tend to be the most specific ones in the context of differentiation of typical manifestations of neuropathological CBD and PSP.

Typical symptoms of PSP are postural instability and falls, cognitive decline, eye movement abnormalities [24] On the other hand CBS is associated with dystonic and myoclonic symptoms, cortical dysfunction and alien limb syndromes, sensory negligence, but also levodopa resistant rigidity $[25,26]$. Interweaving of the symptoms of clinical cases is a challenge in the diagnostic process of some patients. One of the studies showed this combination of symptoms in a patient with premutations in the fragile X mental retardation 1 gene (FMR1) [27]. The case described in that work was symptomatically associated with the clinical manifestation of corticobasal syndrome variant of progressive supranuclear palsy. Symptoms of this patient also included early onset postural instability, echolalia, oculomotor apraxia, increased latency of saccade initiation.

Cognitive impairment and behavioral abnormalities are vital features of both CBS and PSP. Since the description of the disease in 1960s, the aspect of deterioration in cognitive abilities is discussed and perceived as an important factor in the evolution of non-motor symptoms of CBS and PSP. Apathy, disturbances in the executive function, memory and visuospatial deficits are only some factors of progressive symptomatology of $4 \mathrm{R}$ tauopathies [28]. In another study examining patients suffering from PSP or CBS, the assessment of performance of activities of daily living (ADL) led to a conclusion that memory dysfunction is the most important functional disability. The results of ADL analysis presented more disabilities among patients with CBS than PSP [29].

Research conducted by Dutt and coworkers presented the evolution of atrophy in PSP and CBS over 6 and 12 months [30]. The study showed greater longitudinal atrophy rates of cortical and basal ganglia regions among patients with CBS, whereas midbrain and pontine atrophy did not change significantly with time. The study stressed the relevance of $\mathrm{MRI}$ as a tool in combined $4 \mathrm{R}$ tauopathy clinical trials. It also highlighted that PSP rating scale (PSPRS), though not intended to examine CBS, is an element of clinical trial useful in combined $4 \mathrm{R}$ trials due to the multidimensional resemblance of CBS and PSP [30,31].
Differential diagnosis of diseases using imaging methods such as positron emission tomography (PET) and SPECT reveal varied regions of hypometabolism or decreased blood flow and denervation [32]. I-FP-CIT-SPECT and F-Dopa PET present symmetric striatal dopaminergic denervation in patients with PSP and asymmetric striatal dopaminergic denervation in patients with CBS. 99mTc-HMPAO-SPECT/FDG PET shows hypometabolism in frontal lobe and midbrain and asymmetric hypometabolism in striatum and parietal cortex. I-BZM differentiates symmetric in PSP with asymmetric in CBD postsynaptical striatal degeneration.

CBS and PSP have also been analyzed in Polish literature. It had been shown that CBS clinical presentation may become a clinical manifestation of not only PSP pathology, but also AD and Creutzfeldt-Jakob disease [33] Though the review classifies potential morphological changes such as atrophy of midbrain in PSP or cortical atrophy in CBS, imaging of variants with mixed clinical presentation requires further analysis [33]. Multiple mechanisms of pathogenesis of the disease make adequate examination more difficult. It is stressed that in the context of neuroimaging at early stages of CBS, SPECT or PET may be more useful than MRI [33,34]. Usefulness of SPECT and MRI in the context of atypical parkinsonism had been described in 2003 [35]. The study stressed that PSP, CBS and also MSA are the most difficult to differentiate. It also highlighted low sensitivity of the criteria which were then used. [35]. Although new criteria of PSP and CBS have been applied since 2003, the remaining doubtful boundaries of the diseases still create an obstacle in making a proper diagnosis. Multidimensional aspect of PSP and CBS may also reduce the number of differentiating factors.

\section{Conclusion}

Taking into account the background of the disease gained from the interview, physical examination and additional studies, all five patients were diagnosed with a neurodegenerative process with cognitive impairments and a parkinsonian syndrome most likely CBD-PSP. Patients with mixed presentation of symptoms of diseases are difficult to classify to one group. This group of patients represents the most conspicuous part of the analysis concerning primary tauopathies with combined symptomatology. Further examination requires extended assessment of a larger group of patients.

\section{Conflict of interest}

There is no conflict of interest.

\section{Acknowledgement and financial support}

Research supported by internal funds. 


\section{Appendix A. Supplementary data}

Supplementary material related to this article can be found, in the online version, at $\mathrm{d}^{* * *}$ oi:https://doi.org/10.1016/j.pjnns. 2018.06.002

\section{R E F E R E N C E S}

[1] Zhang Y, Walter R, Ng P, Luong PN, Dutt S, Heuer H, et al. Progression of microstructural degeneration in progressive supranuclear palsy and corticobasal syndrome: a longitudinal diffusion tensor imaging study. PLOS ONE 2016;11(6).

[2] Dickson DW. Neuropathology of non-Alzheimer degenerative disorders. Int J Clin Exp Pathol 2010;3(1):1-23.

[3] Dickson DW, Kouri N, Murray ME, Josephs KA. Neuropathology of frontotemporal lobar degeneration-Tau (FTLD-Tau). J Mol Neurosci 2011;45(November (3)):384-9.

[4] Tsai RM, Boxer AL. Clinical Trials: past, current and future for atypical parkinsonian syndromes,. Semin Neurol 2014;34(April (2)):225-34.

[5] Armstrong MJ, Litvan I, Lang AE, Bak TH, Bhatia KP, Borroni $B$, et al. Criteria for the diagnosis of corticobasal degeneration. Neurology 2013;80-496.

[6] Höglinger GU, Respondek G, Stamelou M, Kurz C, Josephs $\mathrm{KA}$, Lang AE, et al. Clinical diagnosis of progressive supranuclear palsy: the movement disorder society criteria. Mov Disord 2017;32(June (6)):853-64.

[7] Wadia PM, Lang AE. The many faces of corticobasal degeneration. Parkinsonism Relat Disord 2007;13:S336-40.

[8] Whitwell JL, Jack Jr CR, Boeve BF, Parisi JE, Ahiskog JE, Drubach DA, et al. Imaging correlates of pathology in corticobasal syndrome. Neurology 2010;75:1879-87.

[9] Josephs KA. Key emerging issues in progressive supranuclear palsy and corticobasal degeneration. J Neurol 2015;262(March (3)):783-8.

[10] Hassan A, Whitwell JL, Josephs KA. The corticobasal syndrome-Alzheimer's disease conundrum. Expert Rev Neurother 2011;11(November (11)):1569-78.

[11] Ling H, de Silva R, Massey A, Courtney R, Hondhamuni G, Bajaj N, et al. Characteristics of progressive supranuclear palsy presenting with corticobasal syndrome: a cortical variant. Neuropathol Appl Neurobiol 2014;40:149-63.

[12] Williams DR. Parkinsonian syndromes. Continuum (Minneap Minn) 2013;19(October (5 Movement Disorders)):1189-212.

[13] Liscis RM, Srulijes K, Groger A, Maetzler W, Berg D. Differentiation of progressive supranuclear palsy: clinical, imaging and laboratory tools. Acta Neurol Scand 2013;127:362-70.

[14] Kurz C, Ebersbach G, Respondek G. An autopsy-confirmed case of progressive supranuclear palsy with predominant postural instability. Acta Neuropathol Commun 2016;4:120.

[15] Whitwell JL, Jack Jr CR, Parisi JE, Gunter JL, Weigand SD, Boeve BF, et al. Midbrain atrophy is not a biomarker of PSP pathology. Eur J Neurol 2013;20(October (10)):1417-22.

[16] Ling $\mathrm{H}$. Clinical approach to progressive supranuclear palsy. J Mov Disord 2016;9(1):3-13.

[17] Yoshida M. Astrocytic inclusions in progressive supranuclear palsy and corticobasal degeneration. Neuropathology 2014;34:555-70.
[18] Litvan I, Hauw JJ, Bartko JJ, Lantos PL, Daniel SE, Horoupian DS, et al. Validity and reliability of the preliminary NINDS neuropathologic criteria for progressive supranuclear palsy and related disorders. J Neuropathol Exp Neurol 1996;55:97-105.

[19] Nukina N, Quan Y, Nakano I, Otomo E. Widespread tau abnormality in a case of cortico-basal degeneration. (English abstract). Rinsho Shinkeigaku 1992;32:1093-101.

[20] Hauw JJ, Daniel SE, Dickson D, Horoupian DS, Jellinger K, Lantos PL, et al. Preliminary NINDS neuropathologic criteria for Steele-Richardson-Olszewski syndrome (progressive supranuclear palsy). Neurology 1994;44:2015-9.

[21] Komori T, Arai N, Oda M, Nakayama H, Mori H, Yagishita S, et al. Astrocytic plaques and tufts of abnormal fibers do not coexist in corticobasal degeneration and progressive supranuclear palsy. Acta Neuropathol 1998;96:401-8.

[22] Dickson DW, Bergeron C, Chin SS, Duyckaerts C, Horoupian $\mathrm{D}$, Ikeda $\mathrm{K}$, et al. Office of rare diseases neuropathologic criteria for corticobasal degeneration. J Neuropathol Exp Neurol 2002;61:935-46.

[23] Shiozawa M, Fukutani Y, Sasaki K, Isaki K, Hamano T, Hirayama M, et al. Corticobasal degeneration: an autopsy case clinically diagnosed as progressive supranuclear palsy. Clin Neuropathol 2000;19(July-August (4)):192-9.

[24] Kułakowska A, Pyd E, Halicka D, Pogorzelski R, Drozdowski W. Cognitive deficits in progressive supranuclear palsy. Neurol Neurochir Pol 2003;37(Suppl. 5):203-10.

[25] Chahine LM, Rebeiz T, Rebeiz JJ, Grossman M, Gross RG. Corticobasal syndrome five new things. Neurol Clin Pract 2014;(August):304-12.

[26] Gruhho M, Sonies B, Frattali CM, Litvan I. Swallowing disturbances in the corticobasal syndrome. Parkinsonism Relat Disord 2015;21:1342-8.

[27] Paucar M, Beniaminov S, Paslawski W, Svenningsson P. PSP-CBS with dopamine deficiency in a female with a FMR1 premutation. Cerebellum 2016. http://dx.doi.org/10.1007/ s12311-016-0793-X

[28] Burrell JR, Hodges JR, Rowe JB. The corticobasal syndrome and progressive supranuclear palsy: a review. Movement Disorders 2014;29(5):684-93.

[29] Cushing N, Jang J, O'Connor CM, Burrell JR, Clemson L, Hodges JR, et al. Disability in atypical parkinsonian syndromes is more dependent on memory dysfunction than motor symptoms. Parkinsonism Relat Disord 2013;19:436-40.

[30] Dutt S, Binney RJ, Heuer HW, et al. Progression of brain atrophy in PSP and CBS over 6 months and 1 year. Neurology 2016;87. http://dx.doi.org/10.1212/WNL.0000000000003305

[31] Golbe LI, Ohman-Strickland PA. A clinical rating scale for progressive supranuclear palsy. Brain 2007;130:1552-65.

[32] Levin J, Kurz A, Arzberger T, Giese A, Hoglinger GU. The differential diagnosis of atypical Parkinsonism. Dtsch Arztebl Int 2016;113:61-9.

[33] Dąbrowska M, Schinwelski M, Sitek EJ, Muraszko-Klaudel A, Brockhuis B, Jamrozik Z, et al. The role of neuroimaging in the diagnosis of the atypical parkinsonian syndromes in clinical practice. Neurol Neurochir Pol 2015;49(6):421-31. http://dx.doi.org/10.1016/j.pjnns.2015.10.002

[34] Paviour D. Progressive supranuclear palsy. In: Saba L, editor. Imaging in neurodegenerative disorders. Oxford University Press; 2015. p. 294.

[35] Lass P, Sławek J, Derejko M, Dubaniewicz M. [Regional cerebral blood flow single photon emission tomography (SPECT) and magnetic resonance imaging (MRI) may be useful in the diagnosis of patients with cortico-basal degeneration, progressive supranuclear palsy and multiple system atrophy]. Neurol Neurochir Pol 2003;37(Suppl. 5):263-74. 